\title{
Change of Compressiblity at the Glass Transition and Prigogine-Defay Ratio in ZrTiCuNiBe Alloys
}

\author{
K. Samwer,* R. Busch, and W. L. Johnson \\ W. M. Keck Laboratory of Engineering Materials, California Institute of Technology, Pasadena, California 91125
}

(Received 28 August 1998)

\begin{abstract}
The change of the compressibility at the glass transition $T_{g}$ is evaluated from pressure experiments in the liquid and the glassy state of the ZrTiCuNiBe bulk metallic glass forming system. Via the enthalpy recovery method, we derive an increase of $T_{g}$ with pressure of $3.6 \mathrm{~K} / \mathrm{GPa}$. Comparing the changes of the compressibility, the specific heat capacity, and the thermal expansion coefficient at $T_{g}$, we estimate for the first time a Prigogine-Defay ratio in metallic systems. This ratio is about 2.4 for the present alloy and compares well with known nonmetallic glass forming systems. [S0031-9007(98)08212-X]
\end{abstract}

PACS numbers: $62.50 .+\mathrm{p}, 62.10 .+\mathrm{s}$, 64.70.Pf

The development of multicomponent bulk metallic glass forming alloys [1,2] has opened the possibility to study the nature of the glass transition also in metallic systems. Glassy phases have been found in a variety of different systems such as polymers, silica, orientational glasses, spin glasses, etc. [3]. Nevertheless, the basic understanding of the glass transition is still a matter of debate [4]. In recent years a number of viscosity [5,6], anelastic relaxation [7], thermal expansion [8-10], and specific heat capacity measurements $[11,12]$ have been performed on the new multicomponent metallic systems. The results confirm the so-called "strong" nature of the glass forming liquid, which exhibits high melt viscosities and Kohlrausch-Watts-Williams exponents around 0.66 near $T_{g}$. Furthermore, they show a step of the specific heat and thermal expansion coefficient at $T_{g}$. Knowing these second derivates of the Gibbs free energy, one can consider discussing Ehrenfest's relations and the Prigogine-Defray ratio for these materials also $[13,14]$.

In this Letter, we present measurements of the heat flow at the glass transition of $\mathrm{Zr}_{46.25} \mathrm{Ti}_{8.25} \mathrm{Cu}_{7.5}$ $\mathrm{Ni}_{10} \mathrm{Be}_{27.5}$ (Vit 4) alloys which have been processed at different hydrostatic pressures. Differential scanning calorimetry at a fixed rate is used to obtain the enthalpy of the as-quenched alloys as well as of samples that were annealed below $T_{g}$ at different pressures. Thereby, we can estimate the enthalpy change and the change in $T_{g}$ with applied hydrostatic pressure. Using this knowledge for the known temperature dependence of the specific volume, we also calculate the change in volume with pressure and get the change of the compressibility at the glass transition. From there it becomes possible to calculate the Prigogine-Defay ratio with the thermodynamic quantities specific heat, compressibility, and thermal expansion for the first time for metallic systems.

The measurements were performed on Vit 4 bulk metallic glasses produced by cooling the alloy from the liquid into the glassy state in a quartz container. This particular alloy was chosen because no dramatic phase separation in the supercooled or amorphous phase has been observed here, in contrast to other alloys of the $\mathrm{ZrTiCu}$ $\mathrm{NiBe}$ family. The samples $(10 \mathrm{~mm}$ in diameter and several $\mathrm{cm}$ long) were machined down into $6 \mathrm{~mm}$ diameter rods which fit the steel and tungsten carbide (WC) pressure cells used in a previous study [15]. By grinding off the outer surface, any remaining oxide particles from the quartz container were removed. The entire cylindrical sample $(6 \mathrm{~mm}$ diameter and $5 \mathrm{~mm}$ long) is amorphous and can be put under hydrostatic pressure in a press (MTP-14, Tetrahedron Associated, San Diego) at various temperatures and times. The maximum force available is $\sim 2 \times 10^{5} \mathrm{~N}$ up to a temperature of $813 \mathrm{~K}$. The samples can be heated with a rate of $8 \mathrm{~K} / \mathrm{min}$ and cooled with a rate of $30 \mathrm{~K} / \mathrm{min}$. We achieved a maximum pressure of $1.4 \mathrm{GPa}$ reaching the mechanical strength of the WC pressure cell. The glassy samples were heated from ambient temperature under pressure up to $678 \mathrm{~K}$, which is above the glass transition temperature, and held there for several minutes. This way the initial state of the processing procedure was always in the undercooled liquid when a hydrostatic pressure was applied. Keeping the pressure constant, we cooled all samples throughout the glass transition with the same cooling rate. Some of the samples were cooled down only to a fixed temperature $(593 \mathrm{~K})$ below the calorimetrically observed $T_{g}$ and annealed there for $16000 \mathrm{~s}$ under a well defined hydrostatic pressure. No crystallization of any kind was observed in any of the samples. Differential scanning calorimetry (DSC) was performed at ambient pressure in a DSC 7 (Perkin-Elmer) under constant heating rate using a small piece of the processed material.

In several recent papers, it has been shown $[16,17]$ that the relaxation state of glassy material can be monitored by the peak height of the heat flow at the glass transition during heating at a constant heating rate. The enthalpy recovery crossing from a nonergodic to an ergodic state indicates the amount of relaxation that has taken place at $T_{g}$ or below. Figure 1 shows the heat flow signal of three $\mathrm{ZrTiCuNiBe}$ samples cooled down below $T_{g}$ under different hydrostatic pressures as indicated and heated up at ambient pressure with the same heating rate. Within 
the resolution of our DSC signal, we cannot detect any different behavior of the differently processed samples. Note that the three curves are shifted with respect to each other for better guidance. At first, this is a surprising result and indicates that the temperature dependence of the enthalpy for heating at ambient pressure is the same for the alloys cooled down from the supercooled liquid at different pressures. That means that even though the three samples were cooled under different pressures throughout the glass transition, they all froze into the same enthalpic state.

In the next step, we annealed the samples at different constant pressures and temperatures well below the calorimetric transition for several hours after cooling through the glass transition under pressure. From previous papers $[16,17]$, we know that this procedure will bring the sample to an enthalpic state very close to a supercooled liquid, if it is observed on a long time scale. In other words, due to the long annealing procedure the sample relaxes into an enthalpic state that is equivalent to a state obtained after a long time scale cooling rate experiment. The resulting enthalpic state can be quenched in again by a rather fast cooling and monitored by an enthalpy recovery experiment in the DSC. In these enthalpy recovery experiments, the relaxed material is heated with a constant rate $(0.0833 \mathrm{~K} / \mathrm{s}$ in our case) throughout the glass transition. In contrast to an unrelaxed sample, a pronounced endothermic "overshooting" effect is observed that can be quantitatively attributed to the enthalpy difference between relaxed and unrelaxed material at the annealing temperature. Figure 2 shows the heat flow data of three samples that are heated up through the glass transition at ambient pressure and constant heating rate, after annealing at $593 \mathrm{~K}$ for $16000 \mathrm{~s}$ at different constant pressure. For comparison, the result of Fig. 1 for a sample that was

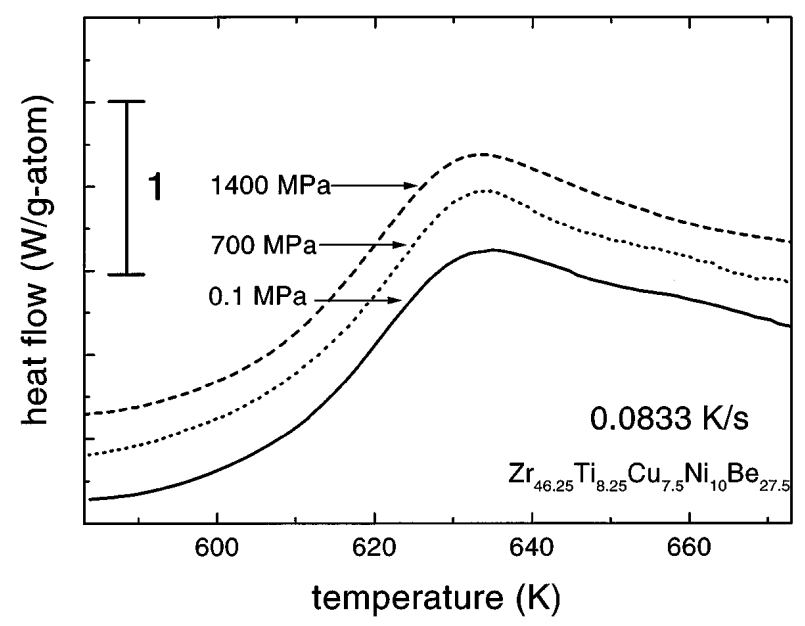

FIG. 1. Heat flow of three ZiTiCuNiBe samples (Vit 4) processed under different hydrostatic pressure upon cooling versus temperature measured at ambient pressure and constant heating rate. cooled at ambient pressure but not annealed below $T_{g}$ is included. As one can clearly see, a small but significant increase in the enthalpy recovery is observed. The higher the hydrostatic pressure, the lower is the enthalpic state that was reached during relaxation. It is important to note that these measurements are done on different pieces from the very same amorphous rod under identical conditions just varying the hydrostatic pressure.

Enthalpy recovery in Vit 4 has been observed previously in annealing experiments under ambient pressure to evaluate the enthalpy dependence of the supercooled liquid for long relaxation times [16]. It was shown that a nearly linear relationship of the enthalpy exists for the extended supercooled liquid versus temperature. In Fig. 3, we introduce the new results for the pressure dependence in that scheme and observe an even larger enthalpy difference for the samples processed under pressure compared to the unrelaxed samples.

Since we did not observe a difference in the enthalpy recovery for those samples cooled under different pressure and no relaxation treatment (Fig. 1), those unrelaxed samples must follow the dotted line in Fig. 3 during cooling. Upon heating, all samples cross over to the solid line at the glass transition according to that particular heating rate at ambient pressure. The reason we do not observe any changes is due to the fact that the supercooled liquid falls out of equilibrium at a slightly higher enthalpy state for higher pressures. This view is corroborated by the relaxation experiments which indeed show a difference in the peak height of the DSC scans. According to the measured enthalpy change upon annealing at $593 \mathrm{~K}$ under different pressures, we shifted the enthalpy curve parallel to the solid line for the supercooled liquid without pressure. This gives us the possibility to determine the shift of $T_{g}$ with pressure.

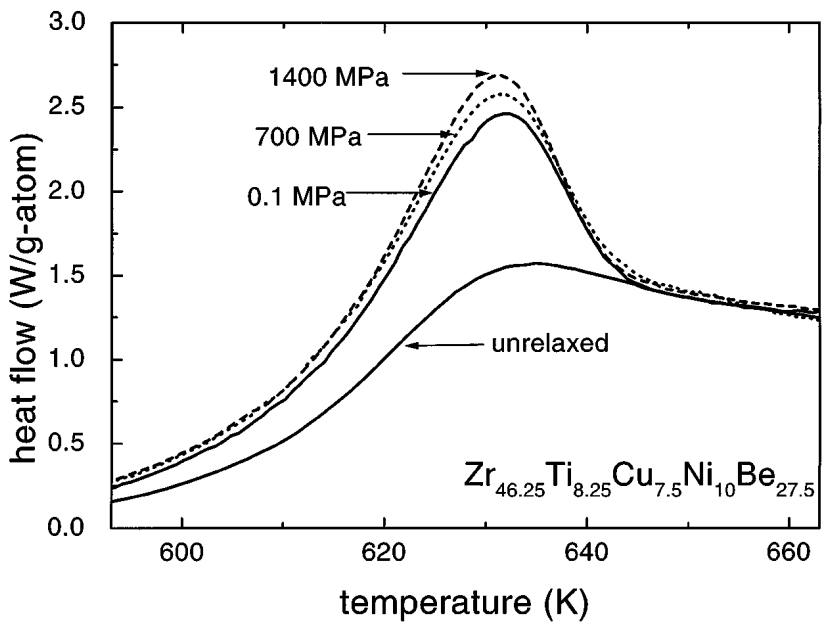

FIG. 2. Heat flow of three different ZrTiCuNiBe samples (Vit 4) relaxed at $593 \mathrm{~K}$ for $16000 \mathrm{~s}$ under different hydrostatic pressures versus temperature measured at ambient pressure and constant heating rate. 


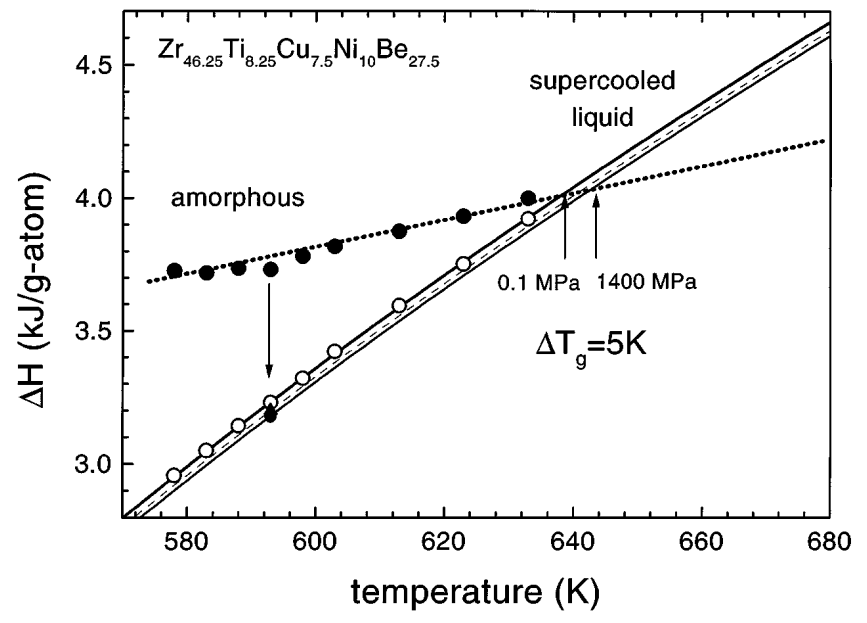

FIG. 3. Enthalpy of the ZrTiCuNiBe system (Vit 4) versus temperature for different states of the alloy. The dotted line represents the glassy (amorphous) phase in the unrelaxed state. The solid line is the representation of the supercooled liquid even below the calorimetric glass transition obtained there by long time relaxation experiments. Both curves are taken from Ref. [16]. The line shifted parallel to the solid line is introduced here according to the pressure-relaxation experiments from this work.

For the glass transition, this measured enthalpy change results in an increase of $T_{g}$ with rising pressure of about $5 \mathrm{~K} / 1.4 \mathrm{GPa}$ or approximately $3.6 \mathrm{~K} / \mathrm{GPa}$. As far as the authors know, the value is measured the first time for metallic systems and compares well with known data of the shift of $T_{g}$ with pressure of other glass forming systems [3].

We were not able to measure the thermal expansion of the same system under hydrostatic pressure in the pressure cell due to the low resolution of our height gauge. To estimate the compressibility change at $T_{g}$ and the PrigogineDefay ratio for these materials, we switched to the relatively close composition $\mathrm{Zr}_{41.2} \mathrm{Ti}_{13.8} \mathrm{Cu}_{12.5} \mathrm{Ni}_{10} \mathrm{Be}_{22.5}$ (Vit 1), where data of the temperature dependence of the specific volume have been published [8]. Figure 4 shows the functional form of these data for the liquid and the glassy states at ambient pressure. Under the assumption that the enthalpy change is proportional to the volume change, the irreversible volume change under $1400 \mathrm{MPa}$ pressure is drawn as a parallel line to the liquid at ambient pressure for the liquid, which is scaled by the observed shift of $T_{g}$ with pressure $(5 \mathrm{~K} / 1.4 \mathrm{GPa})$. Now we can calculate the $\Delta V(p)$ for the liquid state at the glass transition. The change in the compressibility at $T_{g}$ is easily calculated as

$$
\Delta \kappa=-\frac{1}{V} \frac{\Delta V}{\Delta p}=7.85 \times 10^{-14} \frac{\mathrm{m}^{2}}{\mathrm{~N}} .
$$

The compressibility of the glassy state can be estimated by $\kappa^{\text {am }}=[3(1-2 \mu)] / E$, where $E$ equals Young's modulus, and $\mu$ is given by the Poisson ratio. With $E=$ $90 \mathrm{GPa}$ and $\mu=0.354$ [18], we get $\kappa=9.74 \times$

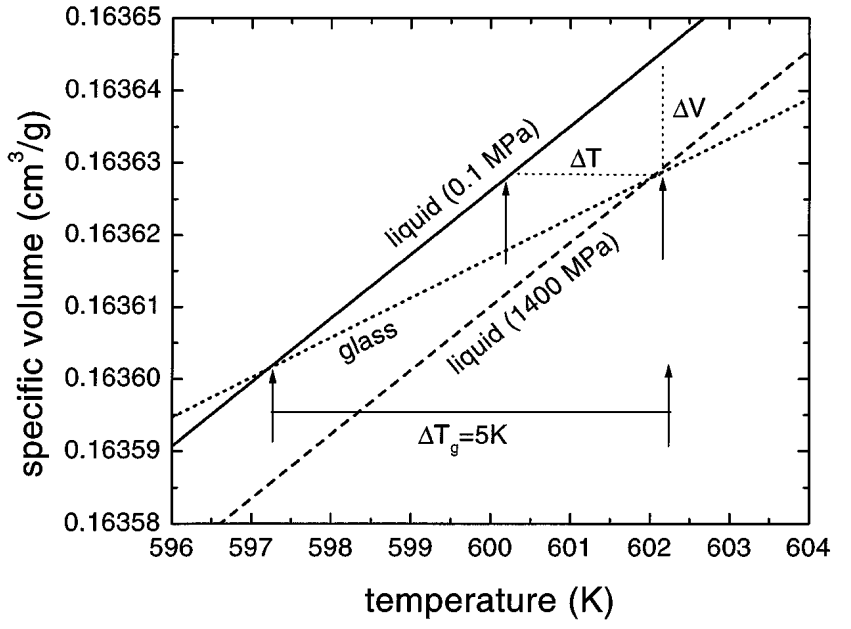

FIG. 4. Specific volume of ZrTiCuNiBe (Vit 1) versus temperature taken for ambient pressure from Ref. [8]. The dashed line for the liquid under $1400 \mathrm{MPa}$ is drawn parallel to the liquid under ambient pressure according to the observed shift of $T_{g}$ with pressure.

$10^{-12} \mathrm{~m}^{2} / \mathrm{N}$. Therefore the change in the compressibility is only $0.8 \%$ at $T_{g}$, which is in accordance with the view that the bulk modulus does not change significantly upon the glass transition because of its relationship with the hard core of the next neighbor potential. The shear modulus, in contrast, changes dramatically with cooling through the glass transition.

Knowing a value for the pressure dependence of the glass transition and the change in compressibility at $T_{g}$, we can go a step further and estimate the Prigogine-Defay (PD) ratio, which is known to be

$$
\mathrm{PD}=\frac{\Delta \kappa \Delta c_{p}}{T_{g} V\left(\Delta \alpha_{p}\right)^{2}}
$$

This ratio is discussed in the literature $[3,13,14]$ by treating the glass transition to be close to a second-order phase transition although the experimentally observable glass transitions are not equilibrium phase transitions. Using the Ehrenfest expressions and the Maxwell relations for the hypothetical equilibrium $T_{g}(p)$ locus defined by volume continuity between the liquid and the glass, one obtains $\left(d T_{g}\right) /(d p)=\left(\Delta \kappa_{T}\right) /\left(\Delta \alpha_{p}\right) \quad$ (first Ehrenfest equation). With the same arguments, one can also discuss the relationship for the entropy changes and obtains the second Ehrenfest equation $\left(d T_{g}\right) /(d p)=$ $\left[T_{g} V\left(\Delta \alpha_{p}\right)\right] /(\Delta c)$ Although the former equation is recently questioned $[13,14]$, we can use the experimentally obtained data for the new bulk metallic glasses and get a PD ratio. The $T_{g}$ is set equal to the Vogel-FulcherTammann temperature $T_{0}=372 \mathrm{~K}$. With a $\Delta c_{p}=$ $42 \mathrm{~J} / \mathrm{g} \cdot$ atom $\cdot \mathrm{K}$ at $T_{0}$ and a $\Delta \alpha_{p}=1.93 \times 10^{-5} \mathrm{~K}^{-1}$, we arrive at a PD ratio of 2.39. Values greater than unity have been frequently observed, but this is the first time the PD ratio for metallic systems has been determined. 
For the interpretation of the PD ratio, one should first recall the way we determined the change in compressibility indirectly from the observed $T_{g}$ shift with pressure. Therefore we cannot contribute to the discussion whether the first or second Ehrenfest relation is incorrect and should be modified. Jäckle [19] corrected the first one and added an extra dependence of the formation volume with pressure, which explains why the Prigogine-Defay ratio is greater than unity. Nieuwenhuizen [14] argues that by correct experimental procedure the first Ehrenfest relation is automatically fulfilled, and the second must be modified by an extra configurational entropy. The experimental work presented here is not able to discriminate among the different interpretations nor whether they might be finally the same if one argues on the change of the configurational entropy and volume with pressure. In the framework of the Prigogine-Defay work, it was argued that more than one order-parameter is responsible for determining the position of equilibrium in a relaxing system. For the glass transition also many dynamic models are discussed recently. Obviously, dynamic heterogeneities in the supercooled liquid play an important role in the heating rate dependence of the glass transition [20,21]. This cannot be analyzed further by this work, where purely thermodynamic arguments were used to give an estimate for the pressure induced variance of the extrinsic variables enthalpy and volume.

In conclusion, we present, for the first time, experimental data for the compressibility change at the glass transition and calculate the Prigogine-Defay ratio for metallic glass forming systems. As is known for nonmetallic systems, the PD ratio measured here is larger than unity. The experiments will be continued for other metallic systems to observe the $T_{g}(p)$ line in a greater variety.

The authors acknowledge a tremendous amount of technical support by U. Bete. The work was supported by the Department of Energy (Grant No. DEFG-0386ER45242).

*Author to whom correspondence should be addressed. Permanent address: Universität Augsburg, Institute für Physik D-86135 Augsburg, Germany.
Email address: samwer@Physik.Uni-Augsburg.DE

[1] A. Inoue, T. Zhang, and T. Masumoto, Mater. Trans. JIM 31, 425 (1990).

[2] A. Peker and W. L. Johnson, Appl. Phys. Lett. 63, 2342 (1993).

[3] For an overview, see papers published in Proceedings of the 3rd International Discussion Meeting on Relaxation in Complex Systems, Vigo, Spain, 1997 [J. NonCryst. Solids (to be published)]; Pablo C. Debenedetti, Metastable Liquids (Princeton University Press, Princeton, NJ, 1996); S. Elliot, Physics of Amorphous Materials (Longman, Essex, England, 1990), 2nd ed., p. 261.

[4] H. Z. Cummings, Gen Li, Y. H. Hwang, C. Q. Shen, W. M. Du, J. Hernandez, and N.J. Tao, Z. Phys. B 103, 501 (1997); C. A. Angell, Curr. Opin. Solid State Mater. Sci. 1, 578 (1996).

[5] E. Bakke, R. Busch, and W. L. Johnson, Appl. Phys. Lett. 67, 3260 (1995).

[6] R. Rambousky, M. Moske, and K. Samwer, Z. Phys. B 99, 387 (1996).

[7] M. Weiß, M. Moske, and K. Samwer, Appl. Phys. Lett. 69, 3200 (1996).

[8] K. Ohsaka, S. K. Chung, W. R. Rhim, A. Peker, D. Scruggs, and W.L. Johnson, Appl. Phys. Lett. 70, 726 (1997).

[9] Y. He and R. Schwarz, J. Non-Cryst. Solids 205, 602 (1996).

[10] N. Geier, M. Weiß, M. Moske, and K. Samwer, J. NonCryst. Solids (to be published).

[11] R. Busch, Y.J. Kim, and W. L. Johnson, J. Appl. Phys. 77, 4039 (1995).

[12] M. Dopfer, B. Reinker, M. Moske, and K. Samwer, Europhys. J. B (to be published).

[13] J. Jäckle, Rep. Prog. Phys. 49, 171 (1986).

[14] Th. Nieuwenhuizen, Phys. Rev. Lett. 79, 1317 (1997).

[15] Eric Bakke, Ph.D. thesis, CALTECH, 1997.

[16] R. Busch and W.L. Johnson, Appl. Phys. Lett. 72, 2695 (1998).

[17] G. Ruitenberg, P. de Hey, F. Sommer, and J. Sietsma, Phys. Rev. Lett. 79, 4830 (1997).

[18] W. L. Johnson, Mater. Sci. Forum 225-227, 35 (1996).

[19] J. Jäckle, J. Cond. Mat. 1, 267 (1989).

[20] R. Brüning and K. Samwer, Phys. Rev. B 46, 11318 (1992).

[21] W. Kob, C. Donati, S. Plimpton, P. Poole, and S. Glotzer, Phys. Rev. Lett. 79, 2827 (1997). 